\title{
Nonlinear Research and Efficient Parameter Identification of Magic Formula Tire Model
}

\author{
Zhun Cheng and Zhixiong Lu \\ College of Engineering, Nanjing Agricultural University, Nanjing 210031, China \\ Correspondence should be addressed to Zhixiong Lu; 1003765165@qq.com
}

Received 6 May 2017; Revised 16 August 2017; Accepted 22 August 2017; Published 28 September 2017

Academic Editor: Stefan Balint

Copyright (c) 2017 Zhun Cheng and Zhixiong Lu. This is an open access article distributed under the Creative Commons Attribution License, which permits unrestricted use, distribution, and reproduction in any medium, provided the original work is properly cited.

\begin{abstract}
The Magic Formula tire model can describe the mechanical properties of tire accurately and thus is applied in the research field of vehicle dynamics widely. The Magic Formula tire model has the characteristics of a great number of parameters and the high nonlinearity, so it is hard to identify parameters. Researchers generally use different intelligent optimization algorithms for parameter identification. However, in the process of parameter identification, with a few experimental data, parameter identification results generally have the low accuracy, while, in the case of a large number of experimental data, the amount of work done in the experiment will increase and there will be many experimental errors. To solve these problems, this paper researches the longitudinal force of tire and proposes an interpolation method and a method based on the nonlinear research of the tire force. The results of parameter identification experiments on the two kinds of tire data show that both of the two methods can be used for the parameter identification of Magic Formula tire model fast and accurately with only a few experimental data. In addition, this paper proposes a method estimating the maximum longitudinal force and corresponding slip rate.
\end{abstract}

\section{Introduction}

Magic Formula tire model is able to well reflect the change rule of the tire force [1-4] and widely used; however, due to the nonlinear property and the required excessive parameters, there are many problems in the current researches on parameter identification of Magic Formula. van Oosten and Bakker [5] proposed that, in order to improve the fitting accuracy, 2000 and 1500 measured data points were needed, respectively, for pure slip the conditions and for combined slip conditions. Cabrera et al. [6,7] and Ortiz et al. [8] proposed calculating the globally optimal solution based on differential evolution algorithm, but it is still likely to fall into the local minimum. Moreover, for the parameter identification of pure longitudinal force, the number of iterations for genetic algorithm is more than 500, and the elapsed time is $180 \mathrm{~s}$. Wang et al. [9] proposed a new self-adaptive differential evolution algorithm (NSADE) and compared with other two differential evolution algorithms IOA and SSPDE; based on NSADE algorithm, the convergence to the global optimum is high in speed, the number of iterations is about 200, and the elapsed time is about $700 \mathrm{~s}$. Zhang et al. [10] proposed a hybrid optimization method, based on which, firstly, the approximate optimal solution is solved according to the genetic algorithm, and then the accurate parameters are identified by the numerical optimization algorithm; the number of iterations for genetic algorithm is 10000; then the calculation results are well fit with the experimental data.

In conclusion, there are some problems in the parameter identification of Magic Formula. If the measured data points are fewer, then the result of the parameter identification is not unique, and there will be many values [11]; due to a small amount of data from the introduced algorithm, many curves may meet the requirements of the objective function; however, for the same tire in the same case, the characteristic curve of the tire force is unique; moreover, the experimental error cannot show and guarantee that several measured data are the highest in accuracy, and the obtained curve is the actual tire force curve. If the measured points are increased, more experimental errors will be introduced, and the workload will be greatly increased. In addition, the interaction of parameter $B, C, D, E$ increases the difficulty in algorithm 
identification; the researchers usually calculate $B, C, D, E$, respectively, and substitute the values into Magic Formula, so the number of the iterations for the algorithm increases, and a great number of data need to be calculated for iteration each time.

In order to solve these problems, this paper proposes an interpolation method and a method based on the nonlinear research of the tire force. The former is based on 9 real experimental types of data under the same kind of load and uses the Lagrange, Hermite, and Spline interpolation to get more experimental data through simulation and then verifies the validity of experimental data obtained through simulation by calculating the errors which may be produced in simulated experimental data. In this way, we can get more experimental data with fewer experiments and then make parameter identification using the genetic algorithm. The method has the high accuracy and a very fast rate of convergence to the optimal solution. The latter, based on the nonlinear characteristic of Magic Formula tire model and the research on the changing laws for longitudinal force of tire, proposes two parameter identification models of $E>$ 1 and $E \leq 1$, respectively, and a method estimating the maximum longitudinal force. The results indicate that under the same kind of load, we can make a fast and accurate parameter identification with a few, sometimes only three, real experimental types of data and get a very accurate estimate of maximum longitudinal force. This paper offers some reference to parameter identifications in other fields.

\section{Parameter Identification of Magic Formula Tire Model Based on Interpolation Method}

The basic Magic Formula of tire longitudinal force is as follows:

$$
\begin{aligned}
Y(x) & =D \sin [C \arctan \{B x-E(B x-\arctan (B x))\}] \\
y(x) & =Y(x)+S_{V} \\
x & =X+S_{H}
\end{aligned}
$$

where $Y$ represents the theoretical longitudinal force of tire; $y$ represents the real longitudinal force of tire; $x$ represents the real slip rate; $X$ represents the theoretical slip rate; parameters $B, C, D, E, S_{H}, S_{V}$ represent the rigidity factor, the shape factor, the peak factor, the curvature factor, the horizontal displacement, and the vertical displacement, respectively. In addition, $B, C, D, E, S_{H}, S_{V}$ are related to the function with the vertical load as the independent variable, so there are secondary parameters [12]. If we can get primary parameters accurately and then we can get secondary parameters easily, and because there are various tire models based on Magic Formula, this paper does not research solving secondary parameters. $S_{H}$ and $S_{V}$ are small, so the research in this paper neglects their values.

The parameter identification method of tire model generally adopts the heuristic intelligent optimization algorithm, like the simulated annealing algorithm, the genetic algorithm, the particle swarm optimization algorithm, and so on. People generally use the coefficient of determination (RSQ) as the objective function of iteration of algorithm. The closer the value of RSQ to 1 is, the higher the accuracy of parameter identification is. The calculation formula of RSQ is as follows:

$$
\begin{aligned}
\mathrm{RSQ} & =1 \\
- & \frac{\sum_{i=1}^{n}\left[F_{x \_ \text {predicted }}\left(x_{i}\right)-F_{x_{-} \text {measured }}\left(x_{i}\right)\right]^{2}}{\sum_{i=1}^{n}\left[F_{x \_ \text {measured }}\left(x_{i}\right)-\left(\sum_{i=1}^{n} F_{x_{\text {_measured }}}\left(x_{i}\right)\right) / n\right]^{2}},
\end{aligned}
$$

where $F_{x_{\text {_p }} \text { predicted }}$ represents the predicted value of longitudinal force of tire; $F_{x_{-} \text {measured }}$ represents the measured value of longitudinal force of tire; $x_{i}$ represents the $i$ th slip rate; $n$ represents the total number of measured values.

The number of experimental data of tire force is the key to parameter identification of Magic Formula tire model. If the number of measured data points is limited, many curves may meet the requirements of objective function, and some curves making the objective function optimal may have strange shapes and thus make the results of parameter identification untrustworthy. Although the tire force has small errors in measurement points, it has considerable errors in some unmeasured points locally; that is, there may be many abnormal points. Substantial data points measured may increase the difficulty and time consumption of experiment and also introduce more experimental errors. With the interpolation method, we can obtain the high-accuracy simulation of experimental data and the proper interpolation method can reduce the error of interpolated data points and the errors of first and second derivatives and then ensure the accuracy of interpolation.

To further ensure the accuracy of interpolation, this paper uses the piecewise interpolation method and each segment is based on 4 measured data points. This is because, according to the changing laws [13], the changes of longitudinal force of tire are generally divided into the following three segments: the first segment approximates to a line; the second segment approximates to a parabola with the slope decreasing first and then increasing; the third segment approximates to a curve with the slope decreasing continuously. Therefore, the parameter identification based on the interpolation method only requires the measurements of 9 types of data under each vertical load.

This paper chooses the measured data of 2 kinds of tire for the research. The first kind of tire's measured data comes from literature [14]; the second kind of tire's measured data comes from ADAMS built-in mdi_pac_94 tire data. The numbers of measured data of two kinds of tire are both 101 under each vertical load. According to the previous analysis, we select 9 measured types of data under the same vertical load as the raw data for interpolation method. Figure 1 shows all measured data and selected 9 measured data types of the first kind of tire. Figure 2 shows all measured data of the second kind of tire.

2.1. Simulating Experimental Data Based on Lagrange Interpolation. According to the previous analysis, we divide the 9 measured data types selected into the following three segments by slip rate: segment one $[0,2,6,8]$, segment two 


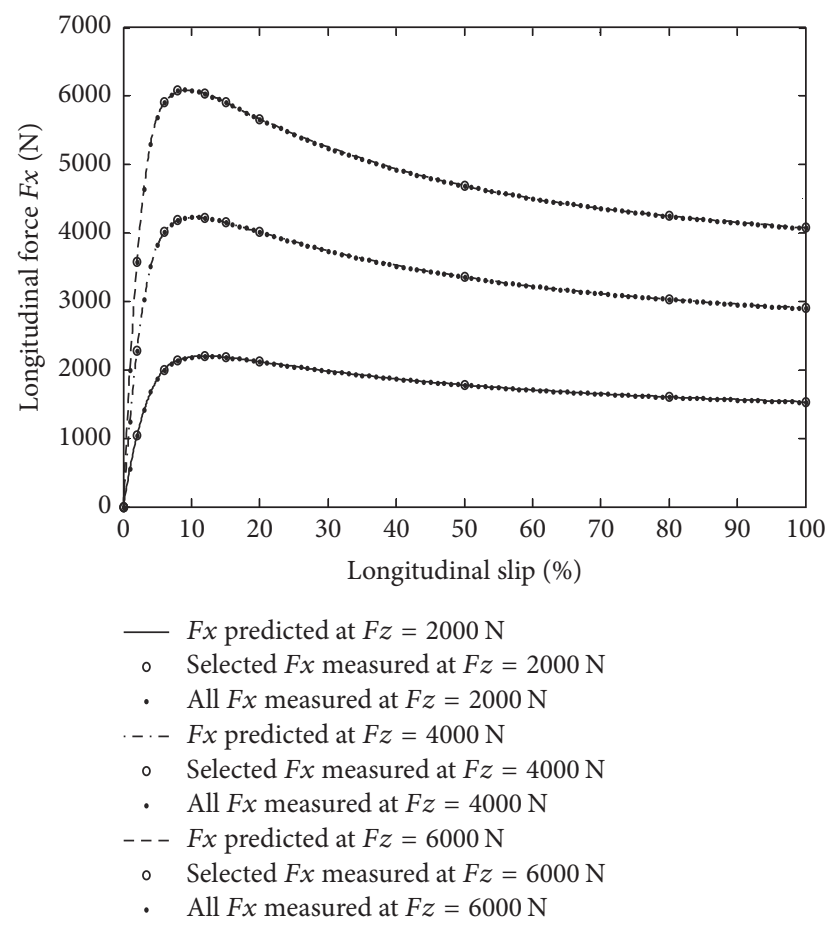

FIGURE 1: The comparison of simulated experimental data and real experimental data of the first kind of tire obtained with the Lagrange interpolation.

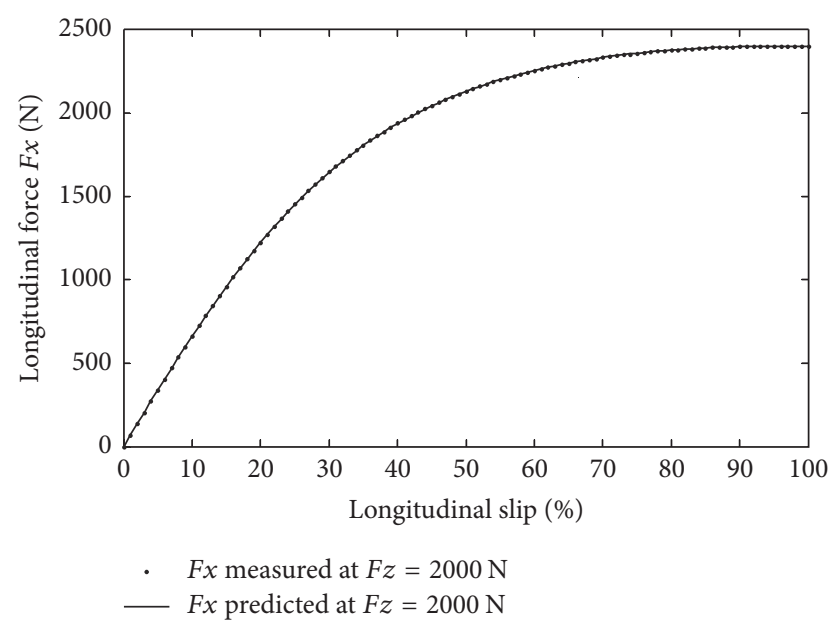

FIGURE 2: The comparison of experimental value and predicted value of the second kind of tire.

$[8,12,15,20]$, and segment three $[20,50,80,100]$. Then, we make the interpolation processing for each segment with the $n$-order Lagrange interpolation polynomial [15-18]. After the processing, each segment has 97 tire force data types obtained through simulation and 4 measured data types. Figures 1 and 2 show the simulated data of tire longitudinal force obtained based on Lagrange interpolation.

Figure 1 shows that the simulated data of interpolation are very close to real experimental data, with the maximum
TABLE 1: The maximum errors of simulated experimental data by Lagrange interpolation method.

\begin{tabular}{lccc}
\hline$F z(\mathrm{kN})$ & \multicolumn{3}{c}{$\operatorname{Slip}(\%)$} \\
& {$[0,2,6,8]$} & {$[8,12,15,20]$} & {$[20,50,80,100]$} \\
\hline 2 & $127.5880 \mathrm{~N}$ & $46.3727 \mathrm{~N}$ & $204.7502 \mathrm{~N}$ \\
4 & $452.0734 \mathrm{~N}$ & $94.3467 \mathrm{~N}$ & $271.1843 \mathrm{~N}$ \\
6 & $1097.03 \mathrm{~N}$ & $129.7200 \mathrm{~N}$ & $264.4633 \mathrm{~N}$ \\
\hline
\end{tabular}

relative error of $6.39 \%$ and the average relative error of $0.499 \%$ which is very small.

Then, we use Lagrange interpolation remainder $R(x)$ to calculate and simulate the maximum error [16-18] which may be reached by experimental data. The estimation formula of error $R(x)$ of Lagrange interpolation is as follows:

$$
|R(x)|=\left|f(x)-P_{n}(x)\right| \leq \frac{M_{n+1}}{(n+1) !}\left|\omega_{n}(x)\right|,
$$

where $x$ represents simulated slip rate data; it is any insertion point in the interval $\left[x_{0}, x_{n}\right] ; x_{i}(i=0,1, \ldots, n)$ is the real slip rate; $f(x)$ is the Magic Formula; $P_{n}(x)$ is the Lagrange interpolation function; $M_{n+1}=\max _{x_{0} \leq x \leq x_{n}}\left|f^{(n+1)}(x)\right| ; \omega_{n}(x)=$ $\left(x-x_{0}\right)\left(x-x_{1}\right) \cdots\left(x-x_{n}\right)$, and $n$ 's value is 3 .

Table 1 shows the maximum errors may be reached by simulated experimental data obtained with Lagrange interpolation. In the table, $F_{z}$ represents the vertical load of tire, and slip represents the slip rate.

Figure 2 shows the results of the data of the second kind of tire with the vertical load of $2000 \mathrm{~N}$ in the same processing mode.

Figure 2 shows that, for the data of the second kind of tire, measured data tally with the experimental data obtained through simulation. The maximum absolute errors may be reached by the simulated data in the first, second, and third segments which are $0.1682 \mathrm{~N}, 0.0958 \mathrm{~N}$, and $168.9035 \mathrm{~N}$, respectively. The data's maximum relative error with real experimental data is $1.08 \%$ and average relative error is $0.13 \%$ which is very small.

2.2. Simulating Experimental Data Based on Hermite Interpolation. In practical problems, the Hermite interpolation [1618] can not only reduce the error between the simulated value and the real value but also reduce the first-order derivative error and thus makes the curve of interpolation have the firstorder smoothness. The estimation formula of error $R(x)$ of cubic Hermite interpolation is as follows:

$$
|R(x)|=\left|f(x)-H_{3}(x)\right| \leq \frac{h^{4}}{384} M_{4},
$$

where $f(x)$ is the Magic Formula, $H_{3}(x)$ is the cubic Hermite interpolation function, $h=\max _{0 \leq i \leq n-1}\left|x_{i+1}-x_{i}\right|, M_{4}=$ $\max _{x_{0} \leq x \leq x_{n}} f^{(4)}(x)$, and $n$ 's value is 3 .

We make the Hermite interpolation processing for the data of the first kind of tire and calculate the maximum error which may be reached by the experimental data obtained through simulation. Table 2 shows the results. 
TABLE 2: The maximum errors of simulated experimental data by Hermite interpolation method.

\begin{tabular}{lccc}
\hline$F z(\mathrm{kN})$ & \multicolumn{3}{c}{$\operatorname{Slip}(\%)$} \\
& {$[0,2,6,8]$} & {$[8,12,15,20]$} & {$[20,50,80,100]$} \\
\hline 2 & $26.5117 \mathrm{~N}$ & $1.9490 \mathrm{~N}$ & $91.3059 \mathrm{~N}$ \\
4 & $106.4405 \mathrm{~N}$ & $4.0898 \mathrm{~N}$ & $117.1631 \mathrm{~N}$ \\
6 & $224.9895 \mathrm{~N}$ & $5.8826 \mathrm{~N}$ & $142.3747 \mathrm{~N}$ \\
\hline
\end{tabular}

TABLE 3: The maximum errors of simulated experimental data by Spline interpolation method.

\begin{tabular}{lccc}
\hline$F z(\mathrm{kN})$ & \multicolumn{3}{c}{$\operatorname{Slip}(\%)$} \\
& {$[0,2,6,8]$} & {$[8,12,15,20]$} & {$[20,50,80,100]$} \\
\hline 2 & $138.8472 \mathrm{~N}$ & $10.0133 \mathrm{~N}$ & $406.1219 \mathrm{~N}$ \\
4 & $591.0820 \mathrm{~N}$ & $20.4681 \mathrm{~N}$ & $528.9091 \mathrm{~N}$ \\
6 & $1343.9231 \mathrm{~N}$ & $28.6259 \mathrm{~N}$ & $644.3526 \mathrm{~N}$ \\
\hline
\end{tabular}

Comparing with the real measured value of tire, the simulated experimental data of the first kind of tire shows the maximum relative error of $3.69 \%$ and the average relative error of $0.82 \%$ which is small.

The second kind of tire's maximum errors may be reached in the first, second, and third segments which are $0.0048 \mathrm{~N}$, $0.0056 \mathrm{~N}$, and $12.7693 \mathrm{~N}$, respectively. Comparing with real experimental data, the data's maximum relative error is $2.05 \%$ and average relative error is $0.18 \%$ which is small.

2.3. Simulating Experimental Data Based on Spline Interpolation. The Spline interpolation [16-18] can meet the higher requirement on smoothness relative to the Hermite interpolation. The method has first-order and second-order continuous differential quotients in the joining point. The estimation formula of error $R(x)$ of cubic Spline interpolation is as follows:

$$
|R(x)|=\left|f(x)-S_{3}(x)\right| \leq e M_{4} h^{4},
$$

where $f(x)$ is Magic Formula, $S_{3}(x)$ is cubic Spline interpolation function, $e=5 / 384, h=\max _{0 \leq i \leq n-1}\left|x_{i+1}-x_{i}\right|, M_{4}=$ $\max _{x_{0} \leq x \leq x_{n}} f^{(4)}(x)$, and $n$ 's value is 3 .

This paper makes the Spline interpolation processing for the data of the first kind of tire and calculates the maximum errors which may be reached by experimental data obtained through simulation, and Table 3 shows the results.

Comparing with the real measured value of tire, the simulated experimental data of the first kind of tire has the maximum relative error of $5.78 \%$ and the average relative error of $0.55 \%$ which is small.

The second kind of tire's maximum errors may be reached in the first, second, and third segments which are $0.0197 \mathrm{~N}$, $0.0238 \mathrm{~N}$, and $60.3164 \mathrm{~N}$, respectively. Comparing with real experimental data, the data's maximum relative error is $1.08 \%$ and average relative error is $0.13 \%$ which is small.

2.4. Parameter Identification of Magic Formula Tire Model Based on Genetic Algorithm. According to the content above,

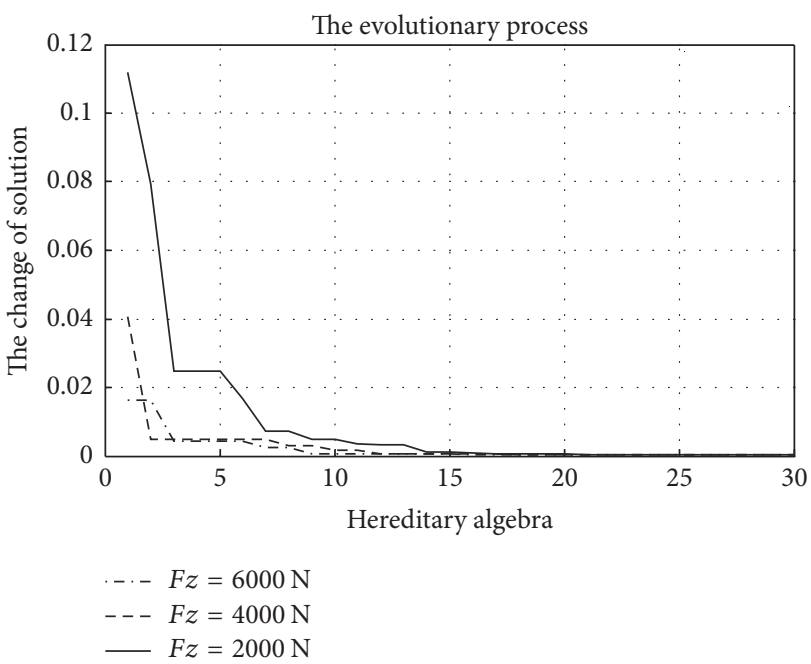

FIGURE 3: Evolution graph of parameter identification of the first kind of tire with genetic algorithm.

the data of tire force obtained based on Lagrange, Hermite, and Spline interpolation almost agree with real data of tire force. Then, we choose the tire force data obtained based on the Lagrange interpolation and use the genetic algorithm [1921] and consider RSQ as the objective function of algorithm iteration to identify the parameters in Magic Formula tire model. The genetic algorithm's initial population is generated randomly, and the number of individuals NIND $=200$ and the hereditary algebra GEN $=30$. Table 4 gives the results of primary parameter identification of the first kind of tire.

Figure 3 shows that the algorithm can get the optimal solution in the 15th generation or so, and the computer consumes the time of about $10 \mathrm{~s}$. Because simulated experimental data are close to real experimental data, the error of any point in the curve can reach the minimum, thus eliminating the possibility of outlier locally. To better compare the results of parameter identification, we compare all measured data (101 data types under the same kind of vertical load) of the first kind of tire with the estimated values of Magic Formula tire mode which have been identified. As shown in Figure 4, the error is very small.

The values of primary parameter identification of the second kind of tire are $B=0.0161, C=1.3635, D=$ $3085.20, E=1.4189$, RSQ $=0.999995$.

\section{Parameter Identification of Magic Formula Tire Model Based on the Nonlinear Research of the Tire Force}

$$
\begin{aligned}
& \text { 3.1. Magic Formula Nonlinear Research. Set } \\
& \qquad F=\arctan \{B x-E(B x-\arctan (B x))\}
\end{aligned}
$$

If $C \cdot F= \pm \pi / 2$, then, in formula (1), $y_{\mathrm{Max}}= \pm D$.

Find the derivative of (1), and attain

$$
\dot{y}(x)=D \cos (C \cdot F) \cdot C \cdot \dot{F} .
$$


TABLE 4: Primary parameter identification results of the first kind of tire.

\begin{tabular}{lccccc}
\hline $\begin{array}{l}\text { Vertical load } \\
F_{Z}(\mathrm{kN})\end{array}$ & $B$ & $C$ & $D$ & $E$ & RSQ \\
\hline 2 & 0.1549 & 1.64 & 2202.97 & 0.5643 & 0.9998 \\
4 & 0.1836 & 1.64 & 4238.65 & 0.5997 & 0.9997 \\
6 & 0.2080 & 1.64 & 6099.19 & 0.5708 & 0.9996 \\
\hline
\end{tabular}

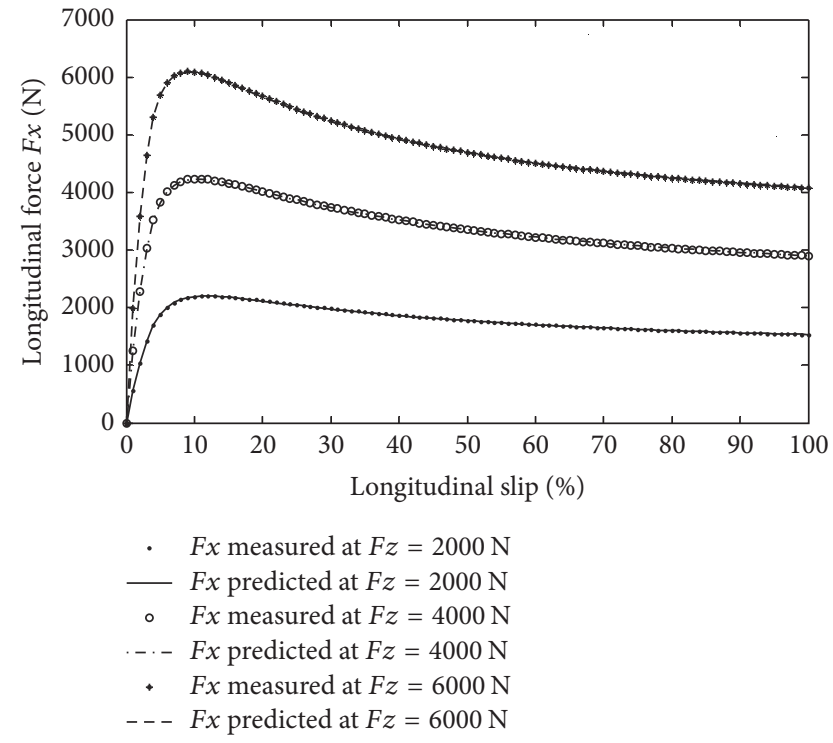

FIGURE 4: Comparison of experimental values and predicted values of the first kind of tire.

Set $\dot{y}(x)=0$; then $\cos (C \cdot F)=0$ or $\dot{F}=0$. Hence, only when $C \cdot F= \pm \pi / 2$ or $\dot{F}=0, \dot{y}(x)$ has the extreme point.

Set $x=0, \dot{y}_{(x=0)}=B C D$. $D$ is the peak factor; when the slip rate $x>0$, assuming $D$ is positive, the slope at the origin is significantly positive, so $B$ has the same positive and negative characteristics as $C$.

$$
\begin{aligned}
f & =B x-E(B x-\arctan (B x)) \\
& =(1-E) B x+E \arctan (B x) .
\end{aligned}
$$

Find the derivative of (10), and attain

$$
\dot{f}=(1-E) B+\frac{E \cdot B}{B^{2} x^{2}+1} .
$$

Find the derivative of (8), and attain

$$
\dot{F}=\frac{1}{f^{2}+1} \cdot \dot{f}
$$

If and only if $x=+\infty, \dot{F}=0$, will the tire longitudinal force generate the extreme point within the scope of the slip rate $[0,100]$; then $\dot{F}=0$; hence, only when $C \cdot F= \pm \pi / 2$, the extreme point must be the maximum point.

If $B>0$, then $C>0$; according to (11), $f$ is monotonically increasing; if $B<0$, then $C<0$, and $f$ is monotonically decreasing. $f$ is the odd function, so the composite function
$F$ is also the odd function; moreover, $F$ and $f$ have the same monotonicity.

$B, C$ must be the same symbol; (8) and (10) are the even functions with respect to $B$, so (1) is the even function with respect to $B, C$, namely, $y_{B>0, C>0}=y_{B<0, C<0}$. Hence, only $B>$ 0 and $C>0$ will be discussed below.

3.1.1. If $E \leq 1 . \dot{f}>0, \dot{F}>0, F$ is monotonically increasing, and $x \rightarrow+\infty, F \rightarrow \pi / 2$. If $C \cdot F=\pi / 2, x \in[0,100]$, then $C>1, y_{\mathrm{Max}}=D$. Assuming the slip rate corresponding to the maximal longitudinal force as $x_{m}$, it is easy to attain

$$
\begin{aligned}
& E=\frac{B x_{m}-\tan (\pi / 2 C)}{B x_{m}-\arctan \left(B x_{m}\right)}, \\
& B=\frac{\dot{y}_{(x=0)}}{C D}=\frac{\dot{y}_{(x=0)}}{C \cdot y_{\text {Max }}} .
\end{aligned}
$$

For $E \leq 1$, as long as $C$ and $\dot{y}_{(x=0)}$ are solved, $x_{m}$ and $\dot{y}_{(x=0)}$ values are measured; then the parameters of Magic Formula can be solved rapidly. Assume $k=\arctan \left(\dot{y}_{(x=0)}\right)=$ $\arctan (B C D)$, due to the character of $\arctan$ function; when the independent variable is greater than 1 , the scope of the dependent variable change is greatly narrowed, so that the independent variable ranges from 40 to 5000 ; the dependent variable, namely, the angle, is only increased by about $1^{\circ}$. Compared with a large number of tire data, the slope range of tangent line for the longitudinal force Magic Formula at the origin is $[40,5729.6]$; the corresponding angle range is $\left[88.57^{\circ}, 89.99^{\circ}\right]$, namely, $k \in[1.5458,1.5706]$.

Combined with the simulated experimental data based on interpolation method mentioned above, as long as the size of longitudinal force from the experimental measured data conforms to the "low-high-low" situation, $x_{m}$ and $y_{\left(x=x_{m}\right)}$ values can be precisely calculated. In the process of identification, select all $k$ values by enumeration method, so that only $C$ is parameter to be calculated, and other parameter values are attained by relation. Theoretically, under each load, as long as three experimental data types are met, the supersimplified parameter identification can be done, because the three data can clearly reflect all information of the tire longitudinal force, such as the values of zero-order, first-order, and second-order derivatives; the experimental data from interpolation simulation can well reflect these rules.

If the measured experimental data cannot meet the "lowhigh-low" requirement or the value span of slip rate is larger, all $x_{m}$ and $k$ values are selected by enumeration method; the parameters to be calculated are $B$ and $C$; other parameter values can be attained by relation. 
TABLE 5: Comparison between the predicted values and measured values of $x_{m}$ and $y_{\left(x=x_{m}\right)}$.

\begin{tabular}{lcccccc}
\hline$F z(\mathrm{kN})$ & $\begin{array}{c}\text { Predicted } x_{m} \\
(\%)\end{array}$ & $\begin{array}{c}\text { Predicted } y_{\left(x=x_{m}\right)} \\
(\mathrm{N})\end{array}$ & $\begin{array}{c}\text { Measured } x_{m} \\
(\%)\end{array}$ & $\begin{array}{c}\text { Measured } y_{\left(x=x_{m}\right)} \\
(\mathrm{N})\end{array}$ & $x_{m}$ error $(\%)$ & $y_{\left(x=x_{m}\right)}$ error $(\%)$ \\
\hline 2 & 12.02 & 2202.75 & 11.9 & 2202.8 & 1.01 & 0.002 \\
4 & 10.02 & 4234.44 & 10.3 & 4235.2 & 2.72 & 0.018 \\
6 & 9.98 & 6090.57 & 9.2 & 6097.2 & 8 & 0.11 \\
\hline
\end{tabular}

TABLE 6: Identification result of the primary parameters for the first kind of tire.

\begin{tabular}{|c|c|c|c|c|c|}
\hline $\begin{array}{l}\text { Vertical load } F z \\
(\mathrm{kN})\end{array}$ & $B$ & $C$ & $D$ & $E$ & RSQ \\
\hline 2 & 0.1539 & 1.64 & 2202.80 & 0.5394 & 0.9999988 \\
\hline 4 & 0.1808 & 1.64 & 4235.20 & 0.5669 & 0.9999893 \\
\hline 6 & 0.2015 & 1.64 & 6097.20 & 0.5509 & 0.9999446 \\
\hline
\end{tabular}

3.1.2. If $E>1$. When $x=0$, then $\dot{f}_{\text {Max }}=B$, and subsequently $\dot{f}$ is monotonically decreasing; when $x \rightarrow+\infty$, then $\dot{f}_{\mathrm{Max}} \rightarrow$ $(1-E) B<0$; hence, $\dot{f}$ is positive firstly and then negative.

Hence, $F$ is firstly increased to the maximum and decreased; three cases are discussed respectively; when $x \in$ $[0,100]$, discuss the relationship between $C \cdot F$ and $\pi / 2$. In Case I, if $C \cdot F_{\mathrm{Max}}=\pi / 2$, then the solution process of the parameters is similar to that of $E \leq 1$; in Case II, if $C \cdot F_{\mathrm{Max}}>$ $\pi / 2$, then there will two maximums, and significantly they do not conform to the actual situation of the tire force, so this case is eliminated; in Case III, if $C \cdot F_{\mathrm{Max}}<\pi / 2$, then $y_{\text {Max }}<D$. Assume $\dot{f}=0, x_{m}=\sqrt{1 /(E-1)} \cdot 1 / B$. The calculated parameter values are as follows:

$$
\begin{aligned}
& E=1+\frac{1}{\left(B x_{m}\right)^{2}}, \\
& D=\frac{\dot{y}_{(x=0)}}{B C} .
\end{aligned}
$$

Therefore, only $B$ and $C$ are the parameters to be calculated; for the calculation of other parameter values refer to the analysis of $E \leq 1$.

3.2. Supersimplified Parameter Identification of Magic Formula. In combination with the study of Magic Formula, based on $B, C, D, E$ nonlinear relationship with respect to the tire force, the supersimplified parameter identification is done. Based on the data for the first and second kind of tire, only three measured data types are selected under each vertical load; the tire force of the measured data needs to meet "low-high-low" requirement; in order to correct the model and select any data point with larger slip rate, a total of four data types are measured, and the parameter identification is based on the genetic algorithm.

The curve close to longitudinal force peak within small scope is approximate to parabola; so Hermite interpolation method is used to predict $x_{m}$ and $y_{\left(x=x_{m}\right)}$.

Table 5 shows the comparison between the predicted values and measured values of $x_{m}$ and $y_{\left(x=x_{m}\right)}$.

For the first kind of tire data, the calculation formulas of $E>1$ and $E \leq 1$ are used, respectively, for parameter identification; when $E>1$, there is no solution, so $E \leq$ 1. $k \in[1.5458,1.5706]$, select 0.0001 as the precision to select all $k$ values within the area, corresponding to the vertical load $F z=2,4,6(\mathrm{kN})$ of the first kind of tire; the optimal value of $k$ is $1.5690,1.5700$, and 1.5703. $C$ is the sole parameter to be calculated by genetic algorithm; as for all the measured data, a total of 101 measured data, all RSQ values are greater than 0.9999; moreover, the optimizing time is about $5 \mathrm{~s}$; the parameter identification results are shown in Table 6.

For the second kind of tire, the calculation formulas of $E>1$ and $E \leq 1$ are used, respectively, for parameter identification; when $E \leq 1$, there is no solution. When $E>$ 1 , the parameter identification result is $k=1.5566, B=$ $0.0173, C=1.2547, D=3197.05, E=1.3545$, RSQ $=$ 0.9987. $x_{m}$ predicted value is 96.99 , the measured value is 97.0 , and the error is $0.01 \% . y_{\left(x=x_{m}\right)}$ predicted value is 2402.42 , the measured value is 2402.42 , and the error is $0 \%$.

The identification results of two tires are consistent with that mentioned above by interpolation method; furthermore, RSQ values of all the measured data are higher.

\section{The Maximal Slip Rate, Maximal Tire Longitudinal Force Prediction, and Parameter Identification}

If three groups of measured data with smaller spacing cannot meet "low-high-low" requirement, it is feasible to predict $k$ and $x_{m}$ value and then identify the parameters. $k \in$ $[1.5458,1.5706]$, select 0.0001 as the precision to select all $k$ values within the area; in each section of $k$ value, make $x_{m}$ vary from 0 to 100 , and substitute it into the derivation formula of the parameter identification, and still select RSQ with respect to all the measured data as the objective function.

When $F z=2000 \mathrm{~N}, x_{m}$ measured value is 11.9. Compared with the predicted value in Table 7 , it can be seen that the slip rate value is closer to $x_{m}$ measured value; the prediction effect will be better. In Table 7 , all the $x_{m}$ measured values are almost less than 20; then compared with the measured value near to the slip rate $20, x_{m}$ value can be obtained accurately. The selection method of $k$ value is in analogy with $x_{m}$ acquisition 
TABLE 7: Predicted results of the first kind of tire under the vertical load $F z=2000 \mathrm{~N}$.

\begin{tabular}{lcc}
\hline Slip rate value $(\%)$ & $x_{m}(\%)$ & $k$ \\
\hline $10,12,14$ & 12 & 1.5690 \\
$20,22,24$ & 12 & 1.5690 \\
$30,32,34$ & 12 & 1.5690 \\
$40,42,44$ & 12 & 1.5690 \\
$50,52,54$ & 16 & 1.5681 \\
$60,62,64$ & 17 & 1.5678 \\
$70,72,74$ & 26 & 1.5653 \\
$80,82,84$ & 14 & 1.5685 \\
$90,92,94$ & 22 & 1.5664 \\
\hline
\end{tabular}

TABLE 8: Predicted results of the second kind of tire under the vertical load $F z=2000 \mathrm{~N}$

\begin{tabular}{lcc}
\hline Slip rate value $(\%)$ & $x_{m}(\%)$ & $k$ \\
\hline $10,12,14$ & 90 & 1.5561 \\
$20,22,24$ & 90 & 1.5561 \\
$30,32,34$ & 92 & 1.5561 \\
$40,42,44$ & 95 & 1.5563 \\
$50,52,54$ & 96 & 1.5563 \\
$60,62,64$ & 97 & 1.5562 \\
$70,72,74$ & 97 & 1.5562 \\
$80,82,84$ & 97 & 1.5565 \\
$90,92,94$ & 97 & 1.5565 \\
\hline
\end{tabular}

method; it can be obtained that $k$ value is 1.5690 and equal to the optimal value of $k$ mentioned in Table 7 .

Table 8 shows the predicted results of the second kind of tire under the vertical load $F z=2000 \mathrm{~N}$.

According to Table $8, x_{m}$ measured value is close to 90 ; find the measured value close to the slip rate 90; then $x_{m}$ predicted value is $97, k=1.5565$, which is almost equal to the measured value 97 and $k$ value 1.5566 mentioned in Table 8 .

After $x_{m}$ and $k$ values are obtained, combine with the measured data for parameter identification, and the process is as mentioned in Section 3.

If only the slip rate $s=[0,10,20,30,40,60,80,100]$ is selected for the first kind of tire data, for convenience of study, only calculate the tire longitudinal force $F x=[0$, 2191.82, 2121.42, 1983.58, 1869.11, 1707.55, 1603.00, 1530.81] when $F z=2000 \mathrm{~N}$. By calculation, $x_{m}=12, k=$ 1.5690 , which are consistent with the previously measured value. In order to compare with the identification result, use the improved simulated annealing algorithm [22-24] for parameter identification.

Table 9 shows the results of two parameter identification methods.

Figure 5 shows the comparison between the predicted values and measured values by new method and simulated annealing algorithm.

It can be seen from Table 9 that the slip rate spacing of the measured points is larger and the quantity is less,

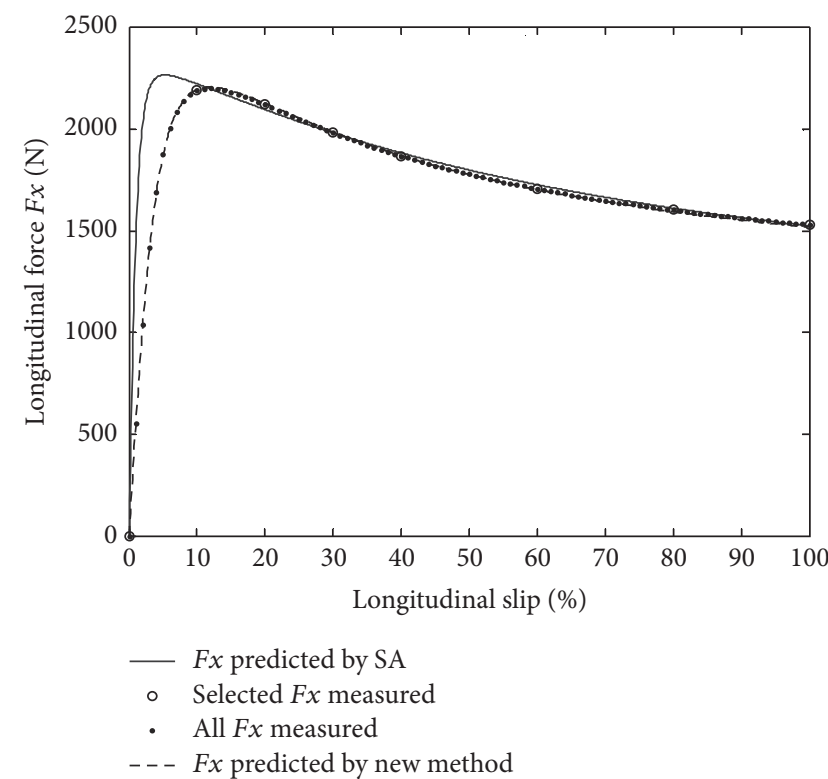

FIGURE 5: Comparison between the predicted values and measured values by new method and simulated annealing algorithm.

if the intelligent algorithm, such as simulated annealing algorithm, is used to guarantee the optimal objective function as the premise, namely, only guarantee the error of the measured points to be minimal; however, for the position beyond the measured points, the error is larger, and RSQ value with respect to a total of 101 measured points is only 0.6970 ; but the slip rate is less than $10 \%$; RSQ value is only 0.4565 . Furthermore, if the identification programs are executed for many times, many groups of parameter solutions will be obtained, and almost these solutions only meet the requirement of 8 measured values; but, in terms of all other measured values, the errors are larger. However, the new method proposed in this paper can accurately calculate the maximal longitudinal force and the corresponding slip rate; moreover, the number of iterations is few; the predicted values by identification are almost equal to all the measured values.

\section{Conclusion}

This paper proposes an interpolation method and a method based on the nonlinear research of the tire force for the parameter identification of Magic Formula tire model. The research shows that the Lagrange, Hermite, and Spline interpolation methods used in this paper can all simulate the experimental data of longitudinal force of tire accurately. According to the changing laws for tire longitudinal force of tire, we divide experimental data into three segments so that we can improve the accuracy of tire force data obtained with interpolation effectively. According to analysis on errors of different interpolation methods, Lagrange, Hermite, and Spline interpolation methods have different ranges of application and are suitable for longitudinal force of tire with simulated slip rates of $20 \% \sim 100 \%, 0 \sim 20 \%$, and $8 \%$ $20 \%$, respectively. Research results indicate that with only 9 
TABLE 9: Results of two parameter identification methods.

\begin{tabular}{|c|c|c|c|c|c|c|c|}
\hline Identification method & $B$ & C & $D$ & $E$ & $\begin{array}{l}\text { Number of } \\
\text { iterations }\end{array}$ & $\begin{array}{c}\text { RSQ (8 } \\
\text { measured } \\
\text { values) }\end{array}$ & RSQ \\
\hline $\begin{array}{l}\text { Simulated annealing } \\
\text { algorithm }\end{array}$ & 0.4466 & 1.7303 & 2267.18 & 0.9094 & 364 & 0.9993 & 0.6970 \\
\hline $\begin{array}{l}\text { Method proposed in this } \\
\text { paper }\end{array}$ & 0.1517 & 1.6624 & 2207.50 & 0.5811 & 3 & 0.9982 & 0.9999 \\
\hline
\end{tabular}

experimental data types under the same kind of vertical load, we can ensure the high accuracy of longitudinal force of tire obtained with interpolation. Based on the tire longitudinal force data obtained with interpolation, we can make the parameter identification of Magic Formula tire model with the genetic algorithm fast and accurately. In the parameter identification process, the number of iterations is 15 and the time consumed by computer is about $10 \mathrm{~s}$. With the method, we not only solve the problem of low accuracy of parameter identification caused by the limited number of experimental data but also reduce the workload in the experiment.

This paper makes the nonlinear research on Magic Formula, deduces the interactive relations of $B, C, D$, and $E$ in Magic Formula, and proposes two kinds of parameter identification models with $E>1$ an $E \leq 1$, respectively, and a method predicting the maximum longitudinal force. Research results show that with only 3 experimental data types under the same vertical load, we can make the parameter identification fast and accurately. The method reduces the number of iterations of parameter identification significantly to only 3 15 iterations which take 3 5 seconds to produce the result on a desktop with Quad CPU $3.4 \mathrm{GHz}$, and the maximum longitudinal force predicted coincides with real experimental data.

\section{Conflicts of Interest}

The authors declare that there are no conflicts of interest regarding the publication of this paper.

\section{Acknowledgments}

This research was supported by the National Natural Science Foundation of China (51175269), National Key Research and Development Plan (2016YFD0701100), and Jiangsu Province Postgraduate Scientific Research Innovation Program (KYCX17_0647).

\section{References}

[1] B. Maclaurin, "Using a modified version of the Magic Formula to describe the traction/slip relationships of tyres in soft cohesive soils," Journal of Terramechanics, vol. 52, no. 1, pp. 17, 2014.

[2] E. Bakker, H. B. Pacejka, and L. Lidner, "A new tire model with an application in vehicle dynamics studies," SAE Technical Papers, 1989.

[3] I. J. M. Besselink, H. B. Pacejka, A. J. C. Schmeitz, and S. T. H. Jansen, "The MF-Swift tyre model: Extending the Magic
Formula with rigid ring dynamics and an enveloping model," Review of Automotive Engineering, vol. 26, no. 2, pp. 245-252, 2005.

[4] H. Olsson, E. Hugon, and C. Rouelle, "Efficient acquisition of tire data and its application in vehicle handling simulation," Tire Science and Technology, vol. 40, no. 4, pp. 234-245, 2012.

[5] J. J. M. van Oosten and E. Bakker, "Determination of magic tyre model parameters," Vehicle System Dynamics, vol. 21, no. 1, pp. 19-29, 1992.

[6] J. A. Cabrera, A. Ortiz, E. Carabias, and A. Simon, "An Alternative Method to Determine the Magic Tyre Model Parameters Using Genetic Algorithms," Vehicle System Dynamics, vol. 41, no. 2, pp. 109-127, 2004.

[7] J. A. Cabrera, A. Ortiz, B. Estebanez, F. Nadal, and A. Simon, "A coevolutionary algorithm for tyre model parameters identification," Structural and Multidisciplinary Optimization, vol. 41, no. 5, pp. 749-763, 2010.

[8] A. Ortiz, J. A. Cabrera, A. J. Guerra, and A. Simon, "An easy procedure to determine Magic Formula parameters: A comparative study between the starting value optimization technique and the IMMa optimization algorithm," Vehicle System Dynamics, vol. 44, no. 9, pp. 689-718, 2006.

[9] Q. Wang, Z. Yang, and K. Ding, "Method in identifying the parameters of magic formula tire model based on new selfadaptive differential evolution," Jixie Gongcheng Xuebao/Journal of Mechanical Engineering, vol. 50, no. 6, pp. 120-128, 2014.

[10] Y. Q. Zhang, W. Chen, L. P. Chen, and J. Z. Yang, "A hybrid optimization on parameters identification for Magic Formula tire model," Automotive Engineering, vol. 29, no. 3, pp. 250-253, 2007.

[11] H. Mitsuhiro, "The development of a parameter identification system for magic formula tyre model," JSAE Review, vol. 16, no. 3, p. 317, 1995.

[12] H. B. Pacejka, Tyre and Vehicle Dynamics, ButterworthHeinemann, Oxford, UK, 3rd edition, 2013.

[13] Z. S. Yu, The Theory of Automobile, Machinery Industry Press, Beijing, China, 2009.

[14] J. Li, ADAMS Practical Tutorial, Beijing Institute of Technology Press, Beijing, China, 2002.

[15] L. Zhang, G. Zhang, and Y.-Q. Qiu, "Research and error analysis of an improved Lagrange interpolation algorithm," Dianli Xitong Baohu yu Kongzhi/Power System Protection and Control, vol. 42, no. 16, pp. 66-70, 2014.

[16] H. Q. Wang and M. L. Lium, Numerical Analysis, Metallurgical Industry Press, Beijing, China, 2004.

[17] Z. Z. Sun, H. W. Wu, W. P. Yuan, and Z. C. Wen, Calculation Method and Practice, Southeast University Press, Nanjing, China, 2009.

[18] M. R. Wang, MATLAB and Scientific Computing, Electronics Industry Press, Beijing, China, 2013. 
[19] C.-W. Chen and Y.-C. Chang, "Support vector regression and genetic algorithm for HVAC optimal operation," Mathematical Problems in Engineering, vol. 2016, Article ID 6212951, 2016.

[20] İ. Kaya and M. Nalbantoğlu, "Simultaneous tuning of cascaded controller design using genetic algorithm," Electrical Engineering, pp. 1-7, 2016.

[21] J. W. Zhuo, Application of MATLAB in Mathematical Modeling, Beihang University Press, Beijing, China, 2011.

[22] Z. Wen, MATLAB Intelligent Algorithm, Tsinghua University press, Beijing, Chnia, 2015.

[23] M. Abdul-Niby, M. Alameen, A. Salhieh, and A. Radhi, "Radhi.Improved genetic and simulating annealing algorithms to solve the traveling salesman problem using constraint programming," Engineering, Technology \& Applied Science Research, vol. 6, no. 2, pp. 927-930, 2016.

[24] Y. Wang, D. Tian, and Y. Li, "An improved simulated annealing algorithm for traveling salesman problem," Lecture Notes in Electrical Engineering, vol. 211, pp. 525-532, 2013. 


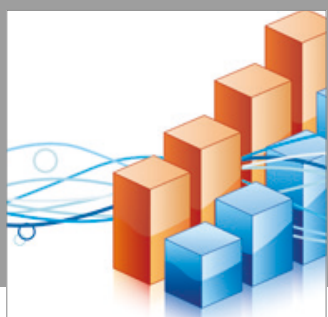

Advances in

Operations Research

vatersals

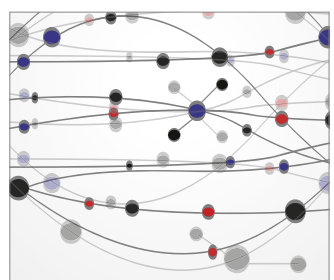

\section{The Scientific} World Journal
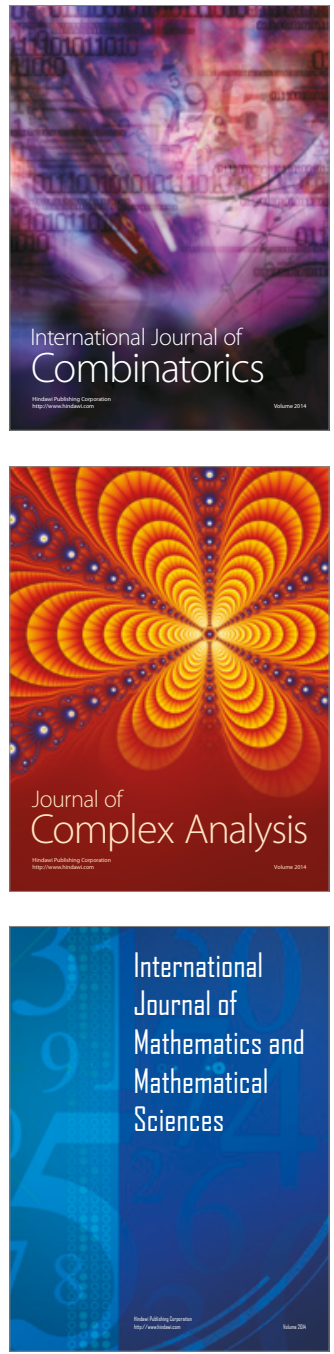
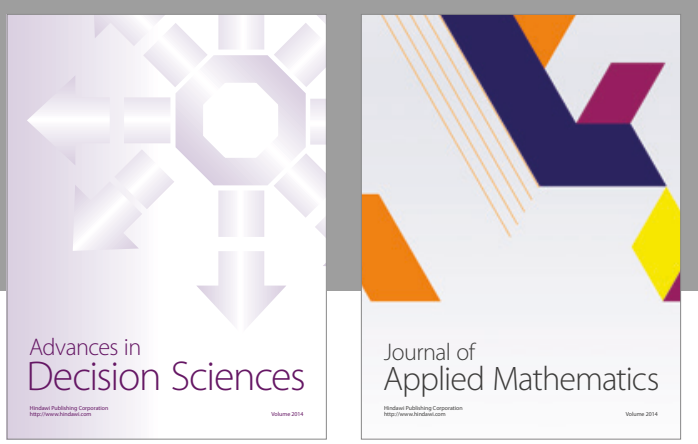

Algebra

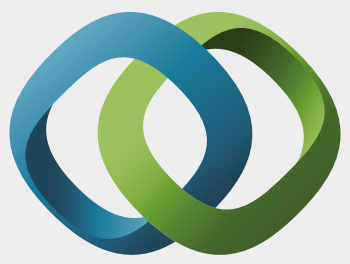

\section{Hindawi}

Submit your manuscripts at

https://www.hindawi.com
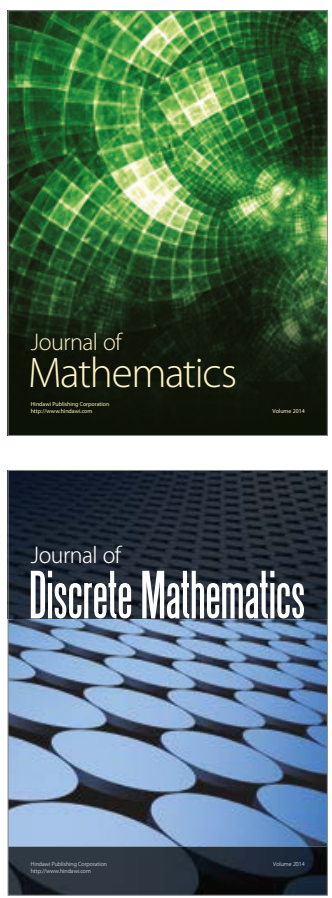

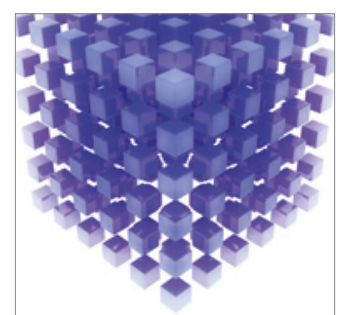

Mathematical Problems in Engineering
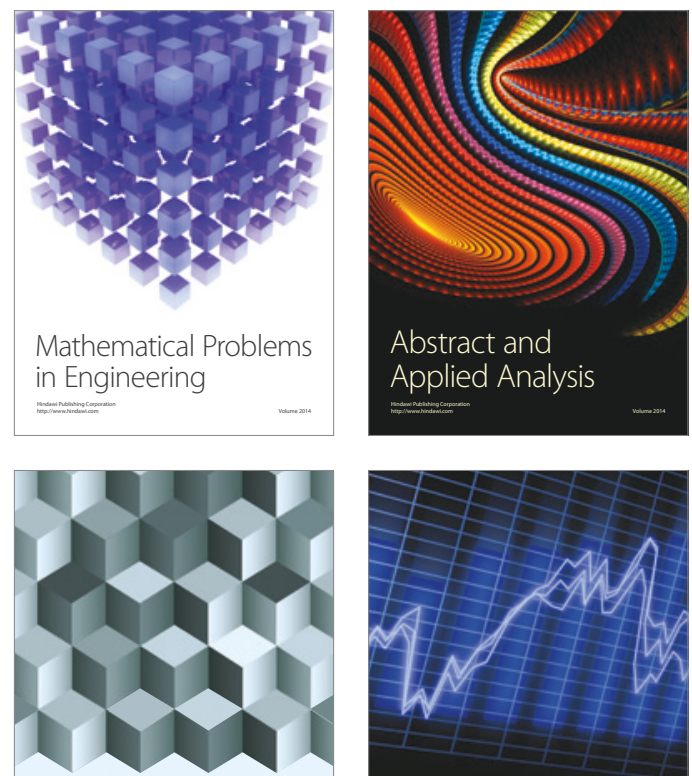

Journal of

Function Spaces

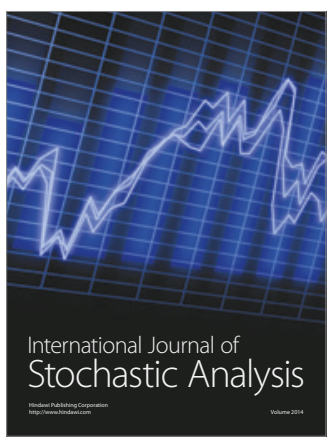

Probability and Statistics
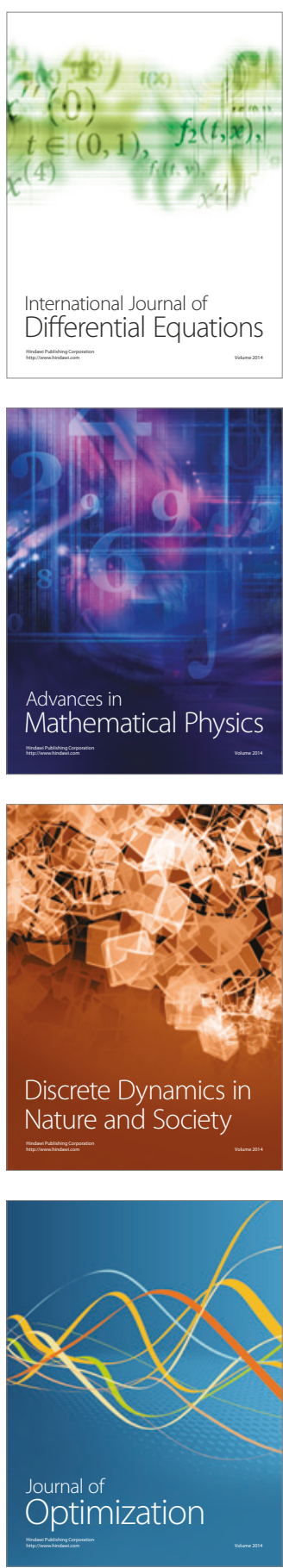